\title{
Contrast based circular approximation for accurate and robust optic disc segmentation in retinal images
}

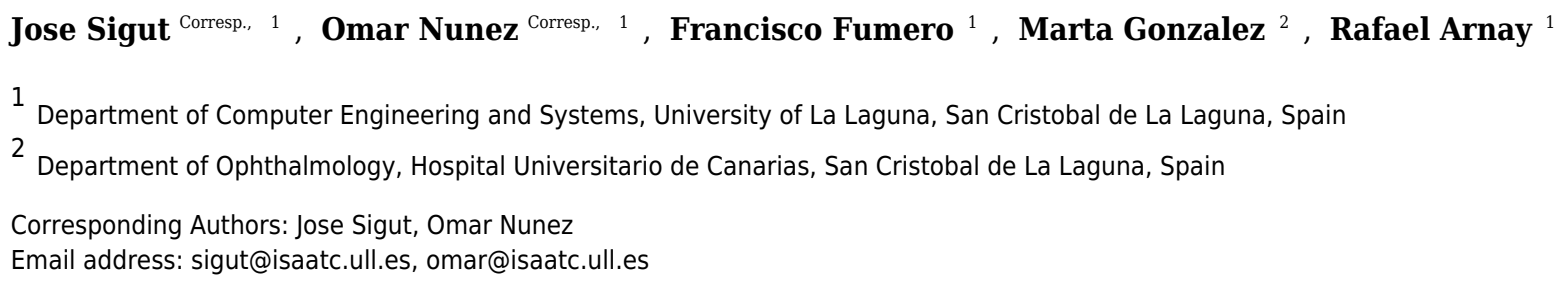

A new method for automatic optic disc localization and segmentation is presented. The localization procedure combines vascular and brightness information to provide the best estimate of the optic disc center which is the starting point for the segmentation algorithm. A detection rate of $99.58 \%$ and $100 \%$ was achieved for the Messidor and ONHSD databases, respectively. A simple circular approximation to the optic disc boundary is proposed based on the maximum average contrast between the inner and outer ring of a circle centered on the estimated location. An average overlap coefficient of 0.890 and 0.865 was achieved for the same datasets, outperforming other state of the art methods. The results obtained confirm the advantages of using a simple circular model under non ideal conditions as opposed to more complex deformable models. 
1 Contrast based circular approximation for accurate and robust optic disc

2

3

7 Corresponding Author:

8 Jose Sigut $^{1}$

9 Facultad de Fisica. Avda. Astrofísico Francisco Sánchez, s/n. 38206. San Cristóbal de La Laguna. Spain

10 Email address: sigut@isaatc.ull.es

11

12

13

14

15

16

17

18

19

20

21

22

23

24 segmentation in retinal images

${ }^{1}$ Department of Computer Engineering and Systems, University of La Laguna, Spain

${ }^{2}$ Department of Ophthalmology, Hospital Universitario de Canarias, Spain

11

3 


\title{
Contrast based circular approximation for accurate and robust optic disc segmentation in retinal images
}

\author{
Jose Sigut $^{1}$, Omar Nunez ${ }^{1}$, Francisco Fumero ${ }^{1}$, Marta Gonzalez ${ }^{2}$ and Rafael Arnay ${ }^{1}$ \\ ${ }^{1}$ Department of Computer Engineering and Systems, University of La Laguna, Spain \\ ${ }^{2}$ Department of Ophthalmology, Hospital Universitario de Canarias, Spain
}

\section{ABSTRACT}

A new method for automatic optic disc localization and segmentation is presented. The localization procedure combines vascular and brightness information to provide the best estimate of the optic disc center which is the starting point for the segmentation algorithm. A detection rate of $99.58 \%$ and $100 \%$ was achieved for the Messidor and ONHSD databases, respectively. A simple circular approximation to the optic disc boundary is proposed based on the maximum average contrast between the inner and outer ring of a circle centered on the estimated location. An average overlap coefficient of 0.890 and 0.865 was achieved for the same datasets, outperforming other state of the art methods. The results obtained confirm the advantages of using a simple circular model under non ideal conditions as opposed to more complex deformable models.

\section{INTRODUCTION}

The optic disc (OD) is one of the main anatomical structures in retinal images. For that reason, its location and segmentation are very important tasks in retinal image analysis and processing. OD analysis is used in the diagnosis of several retinal diseases, most importantly, glaucoma (Haleem et al, 2013) (Almazroa et al, 2015). Moreover, it can be used as a landmark for other retinal features such as the fovea or even as a starting point for vessel tracking.

Localization and segmentation are usually considered as two separate tasks. OD localization is commonly defined as the task of determining the coordinates of a pixel belonging to the optic disc, usually the center. OD segmentation consists of delimitating the disc boundary.

The OD usually appears in retinal images as a bright yellowish region, approximately circular in shape and with blood vessels converging towards its center. These well defined features may give the impression that locating and segmenting the OD is an easy problem. However, very low 
57 contrast images and retinal pathologies such as exudates and different types of lesions turn the

58

problem into very challenging as it is evident by the performance results found in recent publications where the algorithms are tested on large databases.

Many different methods have been proposed in the last years for OD localization and most of them try to take advantage of the basic features mentioned above, the expected appearance of the disc in retinal images and the high density of vessels in the region of interest. Aquino et al. (2010) use three independent detection methods on the green channel to provide three different candidate pixels and a voting procedure is applied to select the best one. In Lu (2011), a circular transformation is designed to simultaneously capture the circular shape of the OD and the image variation across the OD boundary. The center of the OD is determined by the pixels with the maximum variation along multiple radial segments. Giachetti et al. (2014) use vascular and brightness priors based on simple probabilistic detectors, one related to radial symmetry and the other related to vessel density. The work by Mendonça et al. (2013) presents a new technique based on the entropy of vascular directions to assess the convergence of vessels around an image point. Abdullah et al. (2016) use the Circular Hough Transform (CHT) to search for the OD center.

With respect to OD segmentation, most existing methods can be roughly categorized as template-based, deformable model-based, morphology based and, more recently, machine learning methods. In practice, a combination of them is the usual approach. Template-based techniques are mainly based on the assumption of circularity or ellipticity of the OD. Zhu \& Rangayyan (2008), Lu (2011), and Aquino et al. (2010) use the CHT to approximate the OD boundary. Moreover, Aquino et al. (2010) provide an interesting discussion about the convenience of choosing a simple circular approximation as opposed to more complex models. Zheng et al. (2013) and Giachetti et al. (2014) prefer an elliptical approximation. In the work by Giachetti et al., the OD boundary is determined using refined elliptic contours which are finally improved with snake-based algorithms. Regarding the deformable model approaches, Lowell et al. (2004) extract the OD boundary using a global elliptical parametric model with a local deformation. Xu et al. (2007) propose a deformable model which combines active contour deformation with clustering. Yu et al. (2012) use a fast and hybrid level set model with optimized parameters. Mary et al. (2015) propose an OD segmentation scheme by means of the gradient vector flow model. Although these methods can, in theory, provide accurate OD segmentations, they are also very sensitive to artifacts which may be present in the retinal image. Morphology-based techniques primarily make use of the brightness and shape properties of the OD. Reza et al. (2009) use morphological opening, extended maxima operator, and watershed transformation with minima imposition to segment the OD. Welfer et al. (2010) propose a new adaptive morphological method to detect the optic disc center and the optic disc rim. Morales et al. (2013) combine mathematical morphology with principal component analysis. Marin et al. (2015) present a methodology which performs a set of iterative opening-closing morphological operations on an intensity image and the result is thresholded taking also into account blood 
vessel confluence. The OD region is finally obtained by applying the CHT on the output of a Prewitt edge detector. More recently, machine learning methods for OD segmentation have become popular as they provide a powerful tool for feature classification using learned models. Abramoff et al. (2009) perform pixel classification on stereo pairs using an optimal subset of 12 features. To overcome the limitation of classification at the pixel level, Cheng et al. (2013) propose a superpixel based method to segment the OD and the optic cup. Lim et al. (2015) provide a solution based on convolutional neural networks (CNN). Roychowdhury et al. (2016) propose a three-step classification based OD segmentation algorithm. Zilly et al. (2017) present a novel method using ensemble learning based also on $\mathrm{CNN}$ architectures.

In general, the analysis of the literature reveals that most existing methods do not easily handle the problem of the variable appearance of the optic disc in retinal images under non ideal conditions. Model based approaches face the challenge of reliably representing the OD contour and its associated features in the presence of anomalies. Morphology based methods have difficulties in dealing with varying image color, unpredictable intersections of vessels with the OD region and effects of different pathologies. Learning based approaches suffer from the drawbacks inherent to building the appropriate training set. For this reason, a new method for OD localization and segmentation is presented.

The localization procedure combines vascular and brightness information to provide the estimation of the optic disc center which is the starting point for the segmentation task. The segmentation strategy is partly inspired by the work of Aquino et al. (2010) who show that a simple circular model can provide lower overlapping error and better bias-variance trade-off than complex deformable shape/appearance models. As mentioned above, they use the Circular Hough Transform to provide the best approximation to the OD boundary. The approach followed in this work is based on the idea that the average contrast between the outer and inner rings of a circular approximation to the OD boundary is higher than in other parts of the region of interest. By doing so, a much better performance has been obtained as it will be shown in the Results section.

In our opinion, the main contribution of this paper is twofold. First, experimental results show that the simple solution proposed for OD segmentation can outperform other state of the art methods, in particular, in terms of overlap rates. Second, the proposed segmentation method can be used in conjunction with any localization procedure, other than the one suggested in this work, which could even lead to an improvement in the results according to the performance obtained for manual localization.

The rest of the paper is organized as follows. In the Materials \& Methods section, the proposed methodology for OD localization and segmentation is described together with the retinal image databases and the measures which have been used for evaluating the performance. The experimental results which were obtained are presented and compared with other methods in the Results section. The paper concludes with the Discussion and conclusion section. 
MATERIALS \& METHODS

136

137

138

139

140

141

142

143

144

145

146

147

148

149

150

151

152

153

154

155

156

157

158

159

160

161

162

163

164

165

\section{Materials and performance evaluation}

Two publicly available databases of retinal images have been considered to evaluate the performance of the proposed localization and segmentation methods. The main reason for this choice is the widespread use of these datasets and also the availability of a ground truth which can be used in the same conditions for any researcher. There exist other popular databases but the ground truth is not always accessible.

The Messidor database (Decenciere et al., 2014) was created in the framework of diabetic retinopathy screening and diagnosis. It consists of 1200 images acquired in three different ophthalmology departments using a 3CCD color video camera on a Topcon TRC NW6 nonmydriatic retinograph with a $45^{\circ} \mathrm{FOV}$ and three different resolutions: 1440x960, 2240x1488 and $2304 \times 1536$ pixels. This dataset has been used in most of the recent works mainly because of its size, much bigger than other public datasets, and due to the availability of a ground truth with contours traced by an expert from the University of Huelva (University of Huelva, 2012).

The ONHSD database (Lowell et al., 2004) consists of 99 fundus images with a resolution of $760 \times 570$ pixels taken from 50 patients in the context of a diabetic retinopathy screening program. The images were captured using a Canon CR6 45MNf camera with a $45^{\circ} \mathrm{FOV}$. As suggested by Lowell et al, only a subset of 90 images is considered due to the very bad quality of the discarded images. A ground truth is also provided.

In order to evaluate the performance of the proposed methods, different measures are proposed. The OD localization is usually considered as successful if the estimated center lies within the boundary of the corresponding ground truth and that is the approach followed in this work. The error in the localization is evaluated in terms of the error distance, $D\left(c_{\text {exp }}, c_{\text {real }}\right)$, between the real location and the experimental one. Since the size of the retinal images under consideration may be different, a normalized error distance, $D^{*}\left(c_{\text {exp }}, c_{\text {real }}\right)$, becomes a more convenient measure.

$$
D^{*}\left(c_{\text {exp }}, c_{\text {real }}\right)=\frac{D\left(c_{\text {exp }}, c_{\text {real }}\right)}{R}
$$

Where $R$ is the radius of a circle with an area equal to the size of the corresponding ground truth mask.

For the purpose of evaluating the performance of the segmentation method, the Jaccard and Dice coefficients as well as the mean average distance between the true and estimated OD boundaries have been chosen. 
166 The Jaccard and Dice coefficients are measures of the similarity between two sets. The Jaccard

167 coefficient, $J C$, is defined as the ratio between the intersection and union of the automatic

168 segmentation of the OD, $S_{O D}$, and the corresponding ground truth, $S_{\text {Truth }}$.

$$
J C\left(S_{O D}, S_{\text {Truth }}\right)=\frac{\left|S_{O D} \cap S_{\text {Truth }}\right|}{\left|S_{O D} \cup S_{\text {Truth }}\right|}
$$

170

171

The Dice coefficient, $D C$, is defined as twice the ratio between the intersection of $S_{O D}$ and $S_{T r u t h}$, and the sum of their sizes.

$$
D C\left(S_{O D}, S_{\text {Truth }}\right)=\frac{2\left|S_{O D} \cap S_{\text {Truth }}\right|}{\left|S_{O D}\right|+\left|S_{\text {Truth }}\right|}
$$

As opposed to the $J C$ and $D C$ coefficients which are intended for evaluating the degree of overlap between regions, the mean average distance, $M A D$, is used to measure the distances between the closest points on their boundaries. Let us denote by $B_{O D}=\left(a_{1}, a_{2}, \ldots, a_{N}\right)$ and $B_{\text {Truth }}=\left(b_{1}, b_{2}, \ldots\right.$, $b_{M}$ ), the finite sets of points belonging to the boundaries of the segmented OD and the corresponding ground truth, respectively. The $M A D$ between the two sets is defined as follows:

$$
M A D\left(B_{O D}, B_{\text {Truth }}\right)=\frac{1}{2}\left(\frac{\sum_{i=1}^{N} d\left(a_{i,} B_{\text {Truth }}\right)}{N}+\frac{\sum_{j=1}^{M} d\left(b_{j,}, B_{O D}\right)}{M}\right)
$$

Where $d\left(a_{i}, B_{\text {Truth }}\right)={ }_{j}^{\min }\left|b_{j}-a_{i}\right|$ and $d\left(b_{j}, B_{O D}\right)={ }_{i}^{\min }\left|a_{i}-b_{j}\right|$.

It is important to mention that the parameters of the methods for OD localization and segmentation have been experimentally determined using 40 images from the ONHSD dataset. The performance evaluation has been carried out using the whole ONHSD dataset and the Messidor database.

\section{Method for optic disc localization}

The method for the estimation of the optic disc center consists of two main steps: creating a mask based on vascular information to shrink the search space, and filtering the image with a detector which combines vascular and brightness information. The only preprocessing which was found to have a significant impact on the results consisted of resizing the original image using cubic interpolation so that the diameter of the retina becomes equal to 540 pixels as in Marin et al. (2015). By doing so, the proposed methodology can be used without the necessity of adapting the parameters of the algorithms. In the case of using a different angle for capturing the images, the parameters should be readjusted accordingly.

Shrinking the search space using vessel information 
195

196

197

198

199

200

201

202

203

204

205

206

207

208

209

210

211

212

213

214

215

216

217

221

222

223

224

The first step exploits the fact that the OD is the entry point for the major blood vessels that supply the retina. Consequently, the OD region presents usually a high vessel density and can be seen also as a convergence point of this structure. The complementary of the green channel in the RGB color space, $G_{c}$, is taken as the input image for this stage since it is known to provide a good vessel-background contrast. Vessel enhancement, $V_{E}$, is carried out by applying the top-hat operator $T_{8}$ to $G_{C}$ using a disc of 8 pixels in radius as the structuring element.

$$
V_{E}=T_{8}\left(G_{c}\right)
$$

Vessel density, $V_{D}$, is calculated as the difference between the output of two averaging filters on $V_{E}$, an averaging filter of size $80 \times 40$ and an averaging filter of size $80 \times 120$.

$$
V_{D}=A v_{80 \times 40}\left(V_{E}\right)-A v_{80 * 120}\left(V_{E}\right)
$$

The result is normalized dividing by its maximum. The rationale behind this is not only to capture the high density of vessels in the OD region but also take advantage of the fact that the density on both sides of it is significantly lower. A binarized version, $V_{D}^{b}$, is obtained by thresholding $V_{D}$ using a threshold of 0.3 .

The convergence of the branches of the vascular tree, $V_{C}$, is estimated by finding the intersections of lines which are used to approximate the branches of this tree. The lines are the result of the application of the Hough Transform (HT) to the output of a Canny edge detector, $E D_{\text {Canny }}$, computed on the vessel-enhanced image, $V_{E}$, so that the value of each pixel of $V_{C}$ corresponds to the number of Hough line intersections at that point. Due to the noisy nature of this calculation, the resulting image is filtered to obtain an averaged version using a circular averaging filter of radius 40 .

$$
V_{C}=A v_{r=40}\left(H T\left(E D_{\text {Canny }}\left(V_{E}\right)\right)\right.
$$

A binarized version, $V_{C}^{b}$, is obtained by thresholding $V_{C}$ using a threshold of 0.3 .

The two previous masks are combined using a logical $A N D$ operator to provide the final constraint mask, $V_{D C}^{b}$, based on vascular information.

$$
V_{D C}^{b}=\operatorname{logical}_{A N D}\left(V_{D}^{b}, V_{C}^{b}\right)
$$

Fig. 1 shows an example of the different operations involved in the calculation of $V_{D C}^{b}$. It is interesting to remark the role of $V_{C}^{b}$ in the discarding of exudates. Very few lines are detected by the Hough Transform in these unwanted regions as shown in Fig. 1 (e) and (f). 


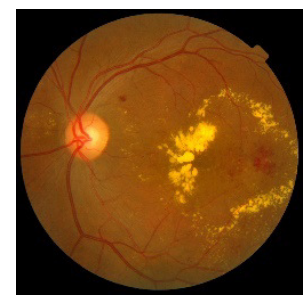

(a)

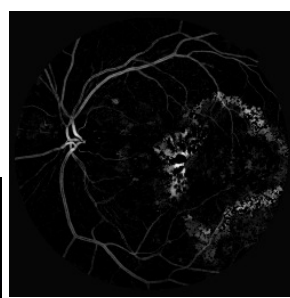

(b)

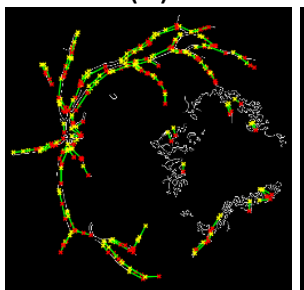

(e)

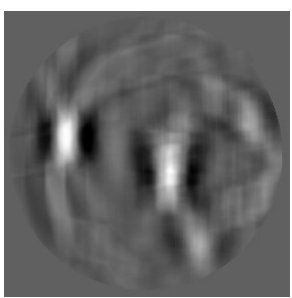

(c)

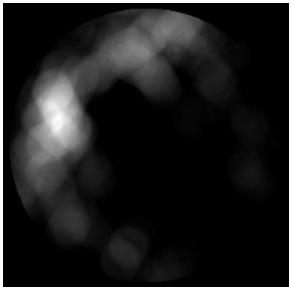

(f)

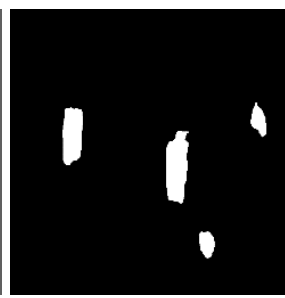

(d)

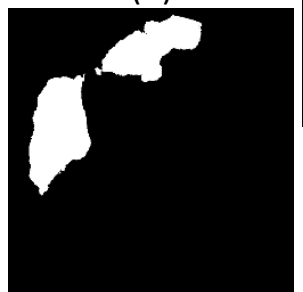

(g)

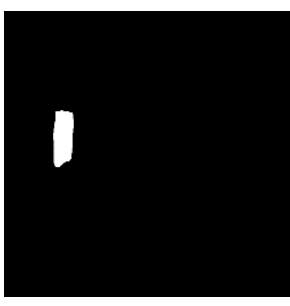

(h)

225 Figure 1 Calculation of the mask based on vessel information. (a) Original image, (b) Vessel enhanced image, (c)

226

227

228

229

230

231

232

233

234

235

236

237

238

239

240

241

242

243
Vessel density, (d) Vessel density mask, (e) Hough Transform of the Canny edge detector output, (f) Vessel convergence, (g) Vessel convergence mask, (h) Logical AND of vessel density and vessel convergence masks

\section{Building the optic disc detector}

The intensity image, $I$, in the second step is calculated as the sum of the three RGB color channels so that the OD usually appears as the brightest region but not always due to artifacts. In fact, the vessel pixels in $I$ which are within the OD may take low values. In order to compensate for this low intensity, a vessel mask, $V_{M}$, is superimposed on the intensity image and the values of the pixels in the mask are set to the maximum value in $I$ to provide the input image, $I_{C}$, for the OD detector. The vessel mask is obtained by thresholding $V_{E}$ to retain only those pixels with high values.

$$
\begin{gathered}
V_{M}=\left\{\begin{array}{cc}
\max (I), & \text { if } V_{E}>\text { percentile }_{98.5}\left(V_{E}\right) \\
0, & \text { otherwise }
\end{array}\right\} \\
I_{c}(p)=\max \left(I(p), V_{M}(p)\right)
\end{gathered}
$$

Where $p$ is any pixel in the image.

The OD detector, $D_{O D}$, is finally calculated as the difference between the output of two averaging filters on $I_{c}$, a circular averaging filter of radius 40 and an averaging filter of size $80 \mathrm{x}$ 160.

$$
D_{O D}=A v_{r=40}\left(I_{c}\right)-A v_{80 * 160}\left(I_{c}\right)
$$


244 The $\mathrm{x}$ and y coordinates where $D_{O D}$ reaches its maximum value after the application of the

245 constraint mask $V_{D C}^{b}$ are taken as the best approximation to the optic disc center. Fig. 2 shows an 246 example of the different operations involved in this second step.

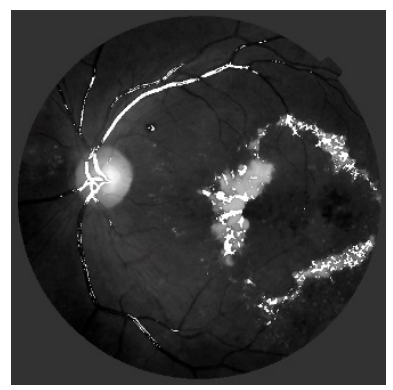

(a)

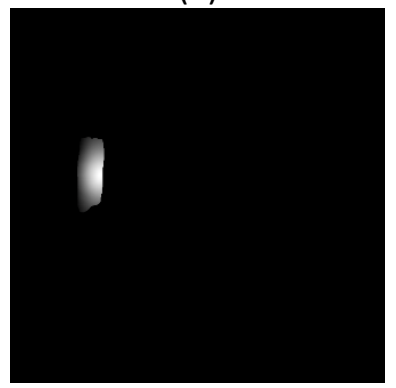

(c)

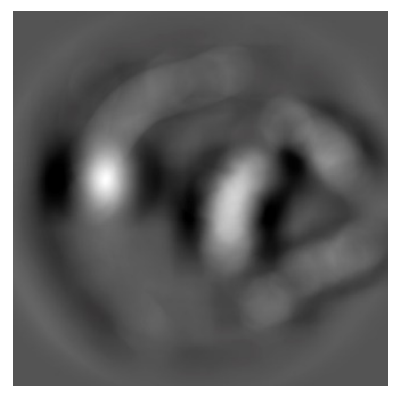

(b)

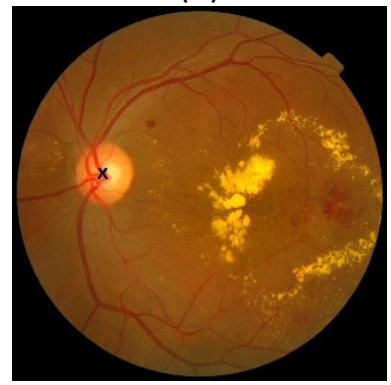

(d)
248

249

250

251

252

253

254

255

256

257

258

259

260

261

262

263

Figure 2 Operations for OD localization. (a) Intensity image with superimposed vessel mask, (b) Output of OD detector, (c) Application of the constraint mask based on vessel information to the output of the OD detector, (d) Estimated OD center

\section{Method for optic disc segmentation}

The method for OD segmentation has been implemented on a subimage of the original RGB retinography by cropping it around the estimated center. The size of the cropping window depends on the resolution of the image under consideration and has been chosen to completely contain the OD and include a certain amount of the background around. In this way, a more robust and efficient segmentation can be achieved due to reduced space for search and consequently reduced number of artifacts present in the whole image.

The intensity image $I$ defined in the previous section is taken as the basis for the calculation of the contrast between the regions of interest. For that purpose, a set of concentric circles were considered with center $\left(x_{c}, y_{c}\right)$, coincident with the center of the window, and increasing radii in increments of $1 \%$, ranging from $25 \%$ to $45 \%$ of the side length of the cropping window. For each reference radius $r$, an outer and inner ring are defined as shown in fig. 3. 


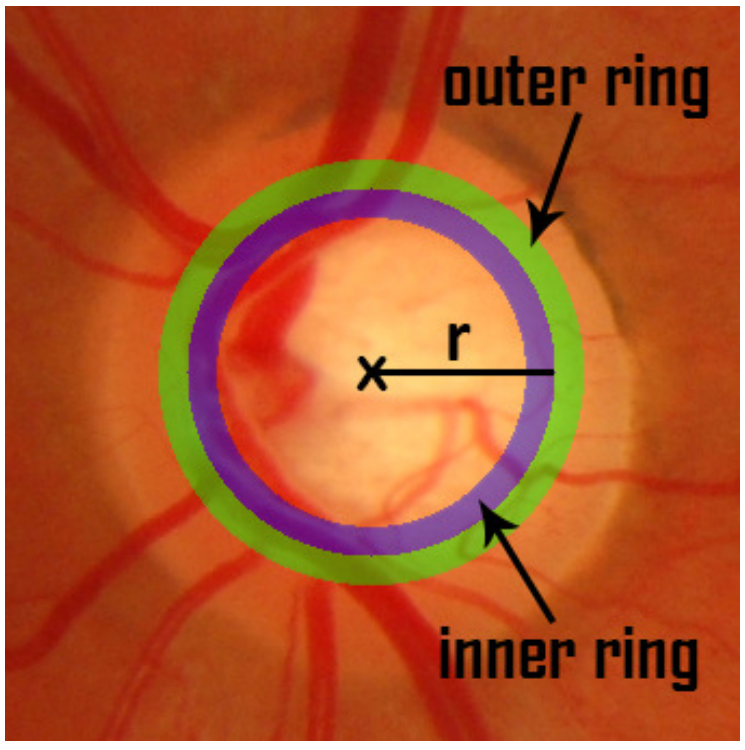

Figure 3 Inner and outer ring for a reference radius $r$

266 The outer ring is the region lying between the two circles with the biggest radii and the inner ring is the region lying between the two circles with the smallest radii. The thickness of the ring was set to $4 \%$ of the side length of the cropping window. A contrast measure is defined as the subtraction of the average intensity in the outer ring from the average intensity in the inner ring:

$$
C M(r)=a v I_{\text {inner }}(r)-a v I_{\text {outer }}(r)
$$

271 The circle for which $C M$ attains the maximum value is taken as the best approximation to the OD 272 contour under these assumptions.

273 We have found that the pure circular approximation obtained in this way may become too rigid 274 in some cases. For this reason, an alternative solution is presented which retains the original 275 simplicity but allowing some more flexibility in the form of considering four fractions of a circle 276 instead as shown in fig. 4. This is the approach which has been followed for OD segmentation.
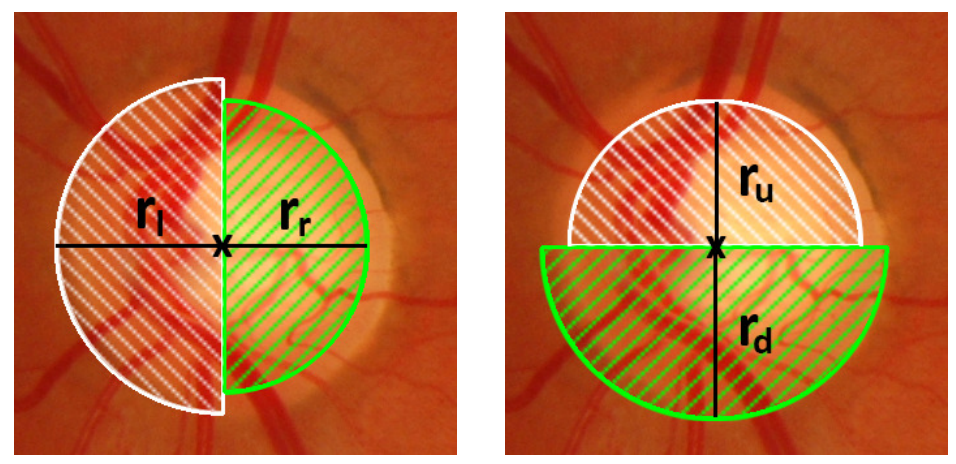
(a)

(b)

279

280

281

282

283

284

285

286

287

288

289

290

291

292

293

294

295

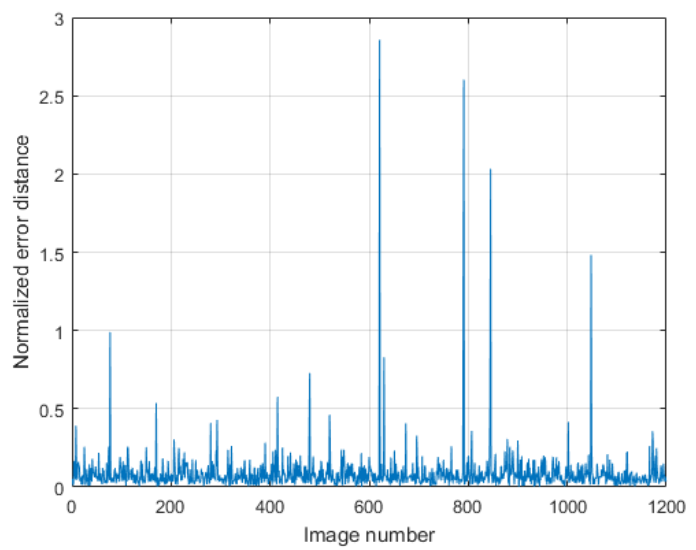

(a)

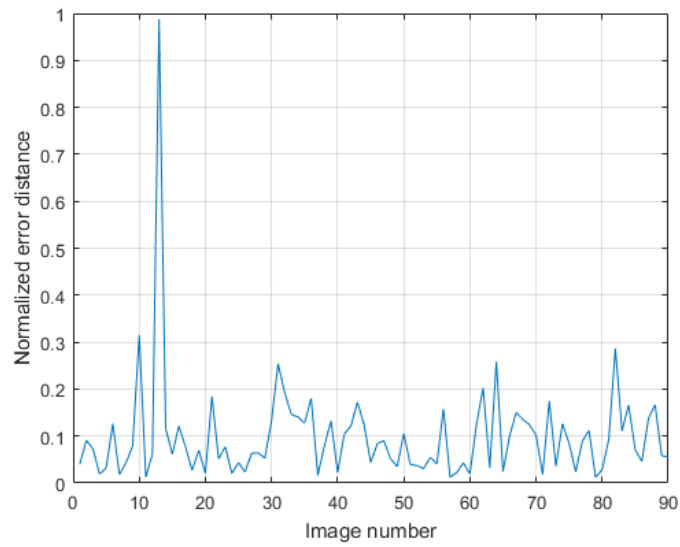

(b) 
Table 1 OD localization accuracy comparison with other methods.

\begin{tabular}{|l|l|l|}
\hline Authors & Database & Accuracy \\
\hline Our method & Messidor & $\mathbf{9 9 . 5 8 \%}$ \\
& ONHSD & $\mathbf{1 0 0 \%}$ \\
\hline Abdullah et al. (2016) & Messidor & $99.25 \%$ \\
\hline Roychowdhury et al. (2016) & ONHSD & $100 \%$ \\
\hline Marin et al. (2015) & Messidor & $100 \%$ \\
\hline Giachetti et al. (2014) & Messidor & $99.75 \%$ \\
\hline Ramakanth et al. (2014) & Messidor & $99.83 \%$ \\
\hline Mendonça et al (2013) & Messidor & $99.42 \%$ \\
\hline Suero et al. (2013) & Messidor & $99.75 \%$ \\
\hline Yu et al. (2012) & Messidor & $99.25 \%$ \\
\hline Sekar \& Nagarakan (2012) & Messidor & $99.58 \%$ \\
\hline Lu (2011) & Messidor & $99.75 \%$ \\
\hline Aquino et al (2010) & Messidor & $98.83 \%$ \\
\hline
\end{tabular}

299

300 Fig. 6 and 7 show some examples of OD localization in the Messidor and ONHSD datasets.

301
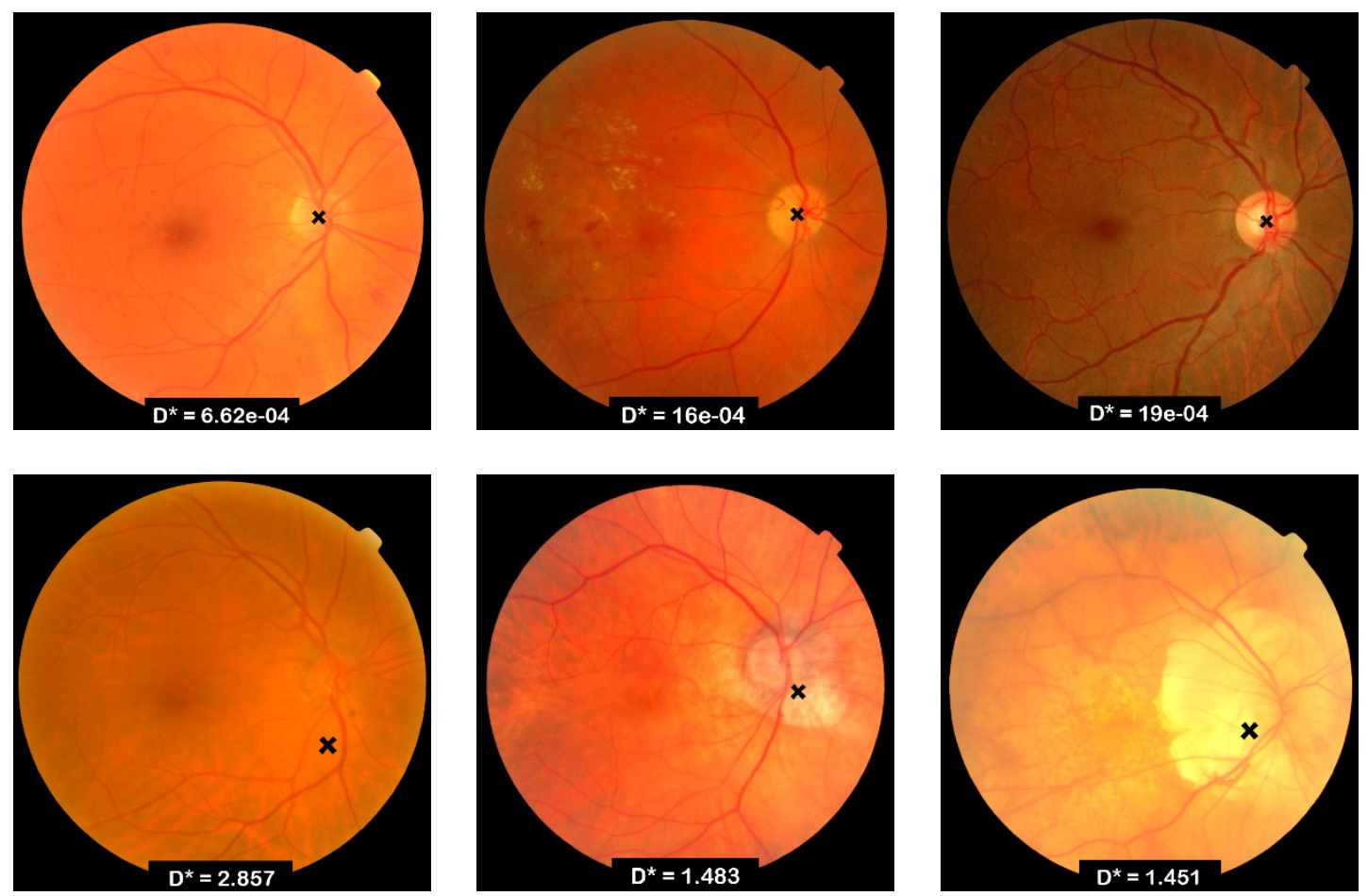

Figure 6 OD localization in images from Messidor database 

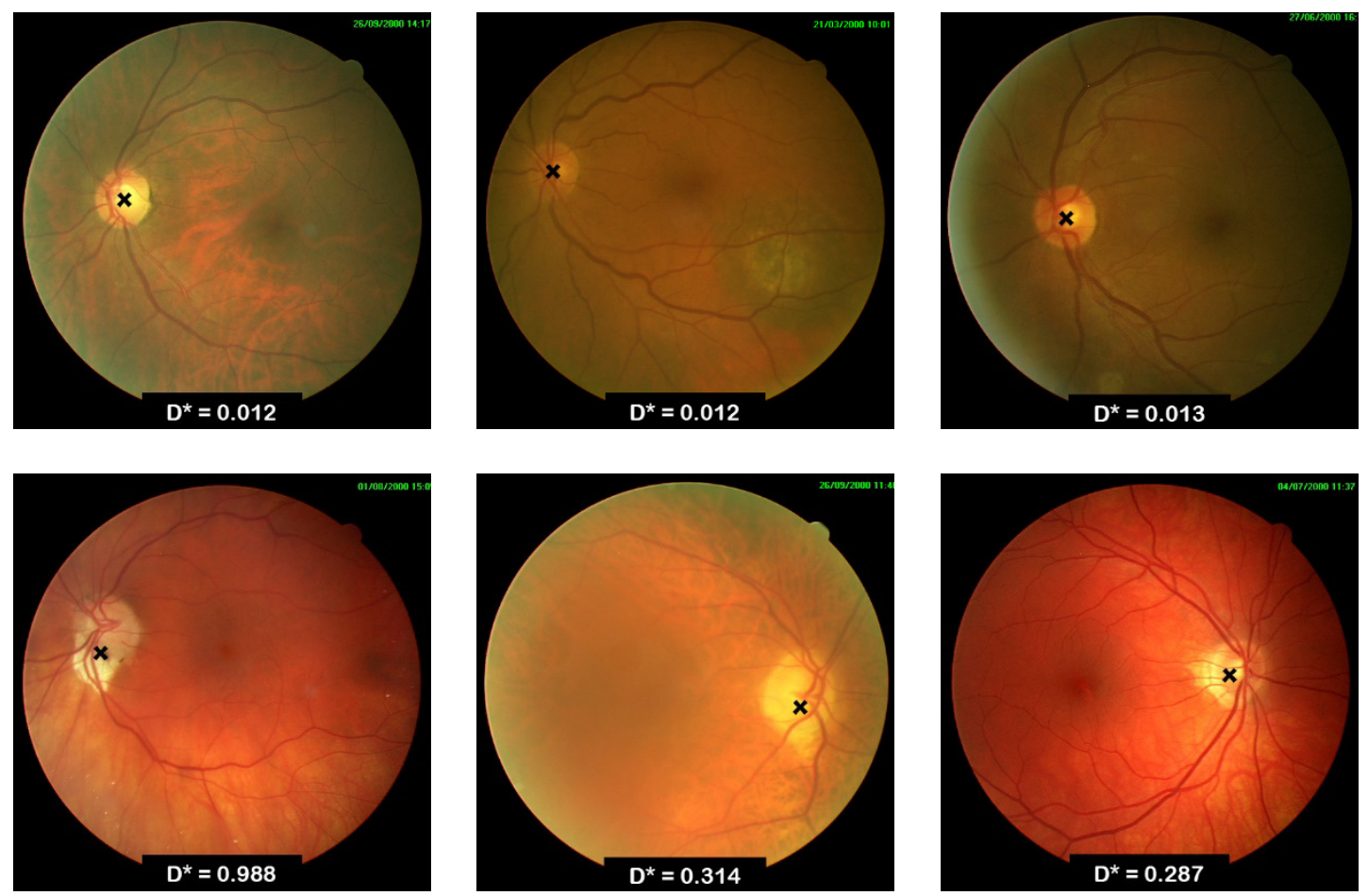

Figure 7 OD localization in images from ONHSD database

\section{Optic disc segmentation}

307

308

309

310

311

312

313

314

315

316

317

318

319

Table 2 shows the performance of our method for optic disc segmentation in terms of the measures described in the materials and performance evaluation section. As it can be seen, two different scenarios have been considered, a completely automatic procedure where the OD center is estimated by means of the localization method explained in this paper and another ideal situation in which the OD center is obtained from the provided ground truth, i.e. manual localization. We have done so to remark the influence of OD localization in the proposed segmentation method and to set a bound on the maximum reachable performance. The average computation time is $1.28 \mathrm{~s}$. per image for the automatic version and $0.07 \mathrm{~s}$. for the manual version so it becomes clear that most of the time is consumed in the localization of the OD. A comparison with other methods has also been included in the table. It should be noted that not all the measures are available for the different methods considered.

Table 2 Performance comparison of OD segmentation methods.

\begin{tabular}{|c|c|c|c|}
\hline Methods & $\overline{J C}$ & $\bar{D}$ & $\overline{M A D}$ \\
\hline \multicolumn{4}{|l|}{ Messidor } \\
\hline Our method (manual localization) & 0.915 & 0.954 & 3.809 \\
\hline Our method (automatic localization) & 0.890 & 0.939 & 5.163 \\
\hline
\end{tabular}




\begin{tabular}{|l|l|l|l|}
\hline Zilly et al. (2017) & 0.90 & - & - \\
\hline Abdullah et al. (2016) & 0.879 & 0.934 & - \\
\hline Roychowdhury et al. (2016) & 0.837 & & 3.9 \\
\hline Dashtbozorg et al. (2015) & 0.886 & 0.937 & 3.160 \\
\hline Lim et al. (2015) & 0.888 & - & - \\
\hline Marin et al. (2015) & 0.87 & 0.92 & 6.17 \\
\hline Giachetti et al. (2014) & 0.879 & - & - \\
\hline Cheng et al. (2013) & 0.875 & - & - \\
\hline Morales et al. (2013) & 0.8228 & 0.8950 & 4.0759 \\
\hline Yu et al. (2012) & 0.844 & - & - \\
\hline Aquino et al. (2010) & 0.86 & - & - \\
\hline & & & \\
\hline ONHSD & & & \\
\hline Our method (manual localization) & $\mathbf{0 . 8 9 3}$ & $\mathbf{0 . 9 4 2}$ & $\mathbf{2 . 2 2 5}$ \\
\hline Our method (automatic localization) & $\mathbf{0 . 8 6 5}$ & $\mathbf{0 . 9 2 4}$ & $\mathbf{2 . 9 1 4}$ \\
\hline Abdullah et al. (2016) & 0.851 & 0.910 & - \\
\hline Dashtbozorg et al (2015) & 0.834 & 0.917 & 2.422 \\
\hline Morales et al. (2013) & 0.804 & 0.887 & 3.275 \\
\hline
\end{tabular}

321 The percentages of images for different intervals of the overlapping coefficient $J C$ are shown in

322

323

324

325

326

327

328

329

330 table 3 as well as a comparison with other methods for the Messidor dataset due to the unavailability of data for ONHSD.

Table 3 Percentages of images for intervals of $\boldsymbol{J C}$ values.

\begin{tabular}{|c|c|c|c|c|c|c|c|}
\hline Methods & $\overline{J C}$ & $J C \geq 0.95$ & $J C \geq 0.90$ & $J C \geq 0.85$ & $J C \geq 0.80$ & $J C \geq 0.75$ & $J C \geq 0.70$ \\
\hline \multicolumn{8}{|l|}{ Messidor } \\
\hline Our method (manual localization) & 0.915 & $23 \%$ & $75 \%$ & $91 \%$ & $96 \%$ & $98 \%$ & $99 \%$ \\
\hline $\begin{array}{l}\text { Our method (automatic } \\
\text { localization) }\end{array}$ & 0.890 & $12 \%$ & $61 \%$ & $84 \%$ & $92 \%$ & $95 \%$ & $97 \%$ \\
\hline Roychowdhury et al. (2016) & 0.837 & - & $20 \%$ & $48 \%$ & - & $83 \%$ & $97 \%$ \\
\hline Dashtbozorg et al. (2015) & 0.886 & $23 \%$ & $66 \%$ & $81 \%$ & $87 \%$ & $92 \%$ & $94 \%$ \\
\hline Lim et al. (2015) & 0.888 & $7 \%$ & $61 \%$ & $86 \%$ & $92 \%$ & $95 \%$ & $96 \%$ \\
\hline Marin et al. (2015) & 0.87 & $12 \%$ & $49 \%$ & - & $84 \%$ & - & $95 \%$ \\
\hline Giachetti et al. (2014) & 0.879 & $13 \%$ & $59 \%$ & $82 \%$ & $88 \%$ & $92 \%$ & $94 \%$ \\
\hline Cheng et al. (2013) & 0.875 & $8 \%$ & $51 \%$ & $76 \%$ & $86 \%$ & $92 \%$ & - \\
\hline Aquino et al. (2010) & 0.86 & $7 \%$ & $46 \%$ & $73 \%$ & $84 \%$ & $90 \%$ & $93 \%$ \\
\hline \multicolumn{8}{|l|}{ ONHSD } \\
\hline Our method (manual localization) & 0.8927 & $10 \%$ & $58 \%$ & $86 \%$ & $93 \%$ & $97 \%$ & $97 \%$ \\
\hline $\begin{array}{l}\text { Our method (automatic } \\
\text { localization) }\end{array}$ & 0.8649 & $4 \%$ & $43 \%$ & $69 \%$ & $87 \%$ & $92 \%$ & $96 \%$ \\
\hline
\end{tabular}

Fig. 8 shows some examples of correct and incorrect segmentations in the Messidor dataset and fig. 9 shows some examples of correct and incorrect segmentations in the ONHSD dataset using the proposed method. 


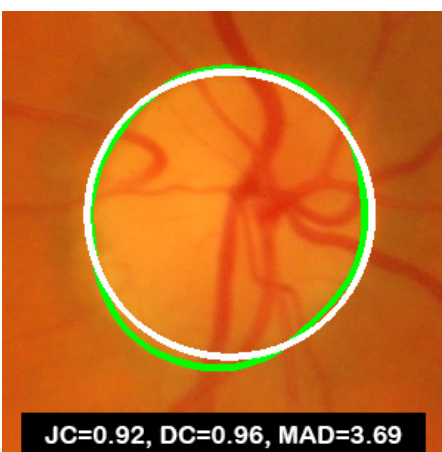

(a)

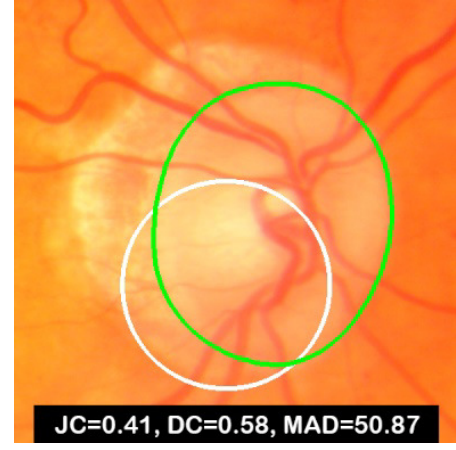

(d)

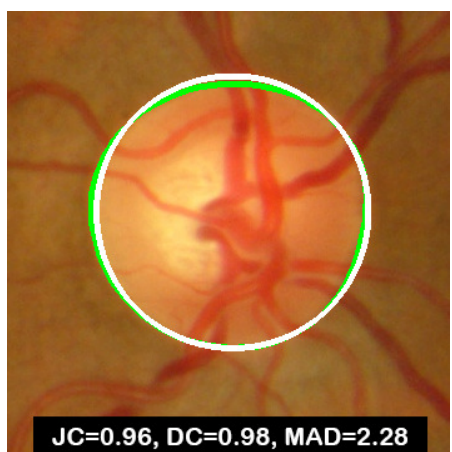

(b)

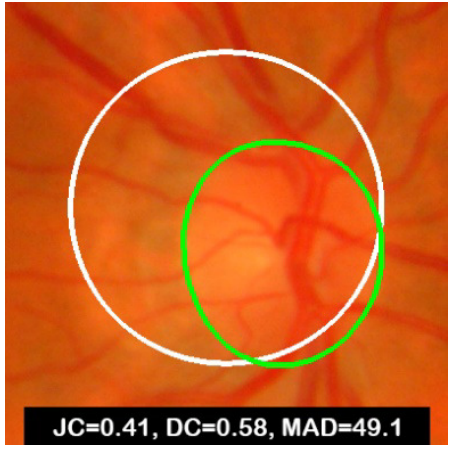

(e)

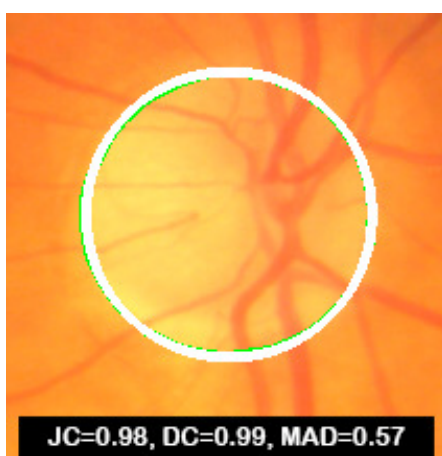

(c)

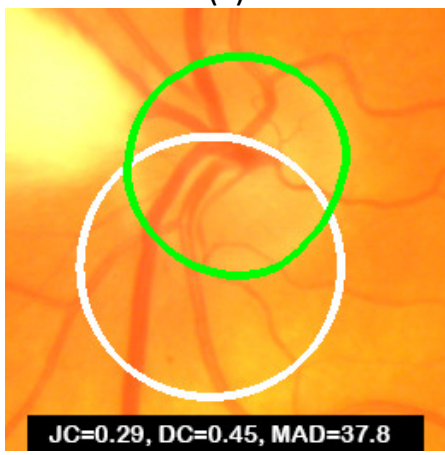

(f)
332

336

337

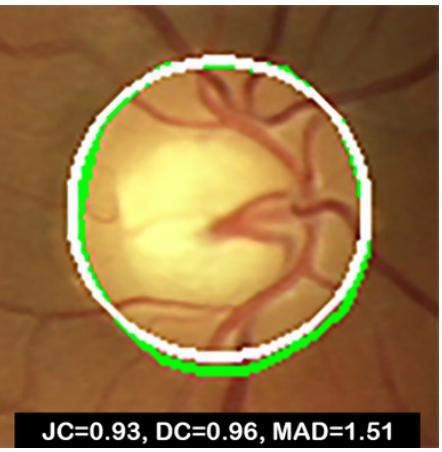

(a)

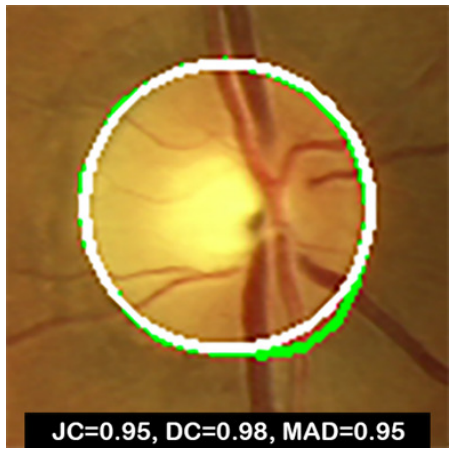

(b)

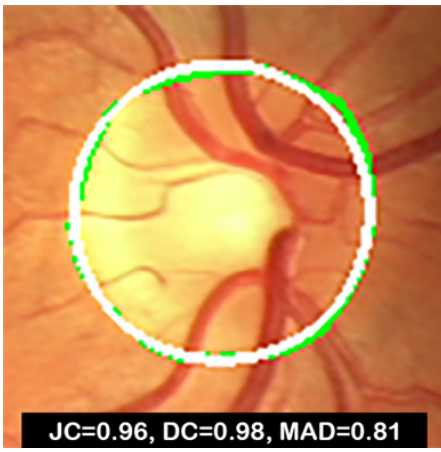

(c) 


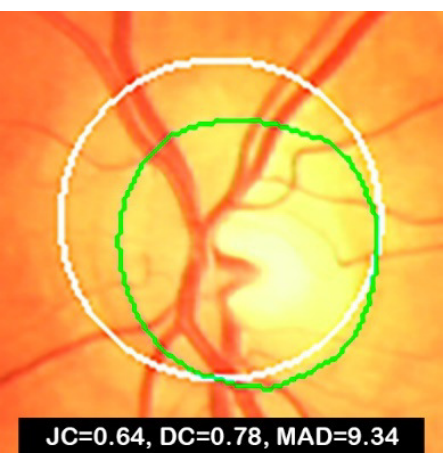

(d)

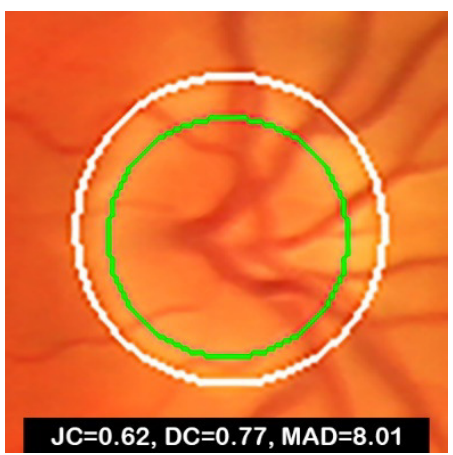

(e)

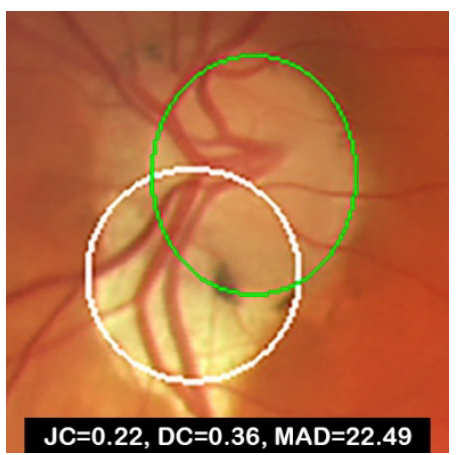

(f)
338

339

340

341

342

343

344

345

346

347

348

349

350

351

352

353

354

355

356

357

358

359

360

361

362

363

364

Figure 9 Images from ONHSD database. (a), (b), (c) Examples of correct segmentations. (d), (e), (f) Examples of incorrect segmentations. The truth OD boundary is marked in green

\section{DISCUSSION AND CONCLUSION}

According to table 1, our results are similar to what has been found with other methods for OD localization. In the case of the Messidor dataset, the detection rate is slightly lower than what has been reported by other researchers. However, these higher rates do not translate necessarily into better segmentation performance as it can be seen in table 2 .

It is important to comment on the expected performance of the algorithms on the range of images found in routine clinical scenarios. Figures 6 and 7 show that the worst results correspond to low contrast images due to the illumination conditions and the presence of peripapillary atrophy. Illumination artifacts can also influence the results negatively, especially if they are located close to the OD. On the other hand, according to the conducted experiments, uneven illumination does not seem to be an issue, nor is it the presence of exudates which is overcome by taking advantage of the output of the Hough line detector as mentioned in the Materials \& Methods section. Choroidal vessels which are very pronounced could lead to wrong detection in localization procedures based on vessel density. The combination of vessel density with the vessel convergence information provided by the output of the Hough detector alleviates the problem. In general, a good performance can be expected as long as the images satisfy the hypothesis of the OD as a bright region with darker surroundings.

Regarding OD segmentation, table 2 indicates that this problem is more difficult than localization. In Giachetti et al (2014), some experiments were conducted to compare automatic and human performance using a subset of 300 images of the Messidor database and three experienced ophthalmologists. Intra and inter-operator average Jaccard coefficients of 0.932 and 0.92 , respectively, were obtained. Therefore, it is clear that there is still some margin for improvement. 
365

366

367

368

369

370

371

372

373

374

375

376

377

378

379

380

381

382

383

384

385

386

387

388

389

390

391

392

393

394

395

396

397

398

399

400

401

402

403

A simple circular model is proposed as the best solution for the OD segmentation problem. The potential theoretical performance of a circular approximation was obtained for each true OD contour by considering circles with varying radius and center coordinates at the center of mass of the corresponding ground truth mask, so that the ones which provided the best scores in terms of $J C$ and $M A D$ were selected. Average $J C$ values of 0.940 and 0.932 were found for the Messidor and ONHSD databases, respectively, and average $M A D$ values of 2.630 and 1.416 were obtained for the same datasets. The results in tables 2 and 3 confirm that in a more realistic scenario, the circular model is still a very good choice. In what follows, we will only consider the segmentation method with automatic localization for the purpose of comparison. To the best of our knowledge, only the method proposed by Zilly et al (2017), using ensemble learning based on CNN, outperforms ours in terms of the average $J C$ on the Messidor dataset. However, their paper is mostly focused on the DRISHTI-GS dataset and hardly any detail is provided regarding the application of their method to Messidor. They claim that the OD is first localized by applying a CHT on the green channel but the obtained success rate is not reported neither is its impact on the results. Moreover, as the authors claim, one of the limitations of their algorithm comes from its non-deterministic behavior because of the sampling of points of the training images. The other methods report worse results for average $J C$ and $D C$ in both databases. With respect to the average $M A D$, our results are not the best but still satisfactory. This is something expected due to the lack of flexibility of the circular model to adapt to some of the true OD boundaries as compared to other models. The performance scores in table 3 also show that our method is rather stable for values of $J C$ ranging from 0.70 to 0.85 and provides, overall, desirable results across all intervals.

The fact that the segmentation method is performed on a subset of the original image reduces the number of artifacts and sources of error. Figures 8 and 9 show that poorer segmentations occur mainly due to low contrast between the inner and outer rings of the OD and, more importantly, because of the strong influence of the accuracy in the estimation of the disc center as it becomes clear from the results obtained. If the distance between the estimated OD center and the ground truth OD center is considerable, the proposed method will fail to produce an acceptable solution. Manual localization provides much better results. Therefore, future work will be focused on decreasing the localization error. Even in the case of perfect localization, there could be an issue with the segmentation of glaucomatous optic discs with high cup to disc ratio given that the maximum contrast could be achieved at the cup boundary according to (12). The confirmation of this point is also left for future investigation since it requires the use of retinal image databases intended for glaucoma diagnosis.

In conclusion, this research work seems to agree with the interesting discussion carried out by Aquino et al. (2010) about the advantages and disadvantages of using circular, elliptical and deformable models. They compared the circular approach with four different elliptical models and three deformable models and concluded that under appropriate background-contrast conditions, the deformable models render more accurate OD segmentations but under not so 
404 ideal conditions, a circular model is preferable because it provides a more robust and reliable 405 solution. It is, therefore, another illustrative example of bias-variance trade-off in model fitting.

406

407

408

409

410

411

412

413

414

415

416

417

418

419

420

421

422

423

424

425

426

427

428

429

430

431

432

433

434

\section{REFERENCES}

Abdullah M, Fraz MM, Barman SA. 2016. Localization and segmentation of optic disc in retinal images using circular Hough transform and grow-cut algorithm. PeerJ 4:e2003. DOI: 10.7717/peerj.2003.

Abramoff MD, Alward WL, Greenlee EC, Shuba L, Kim CY, Fingert JH, Kwon YH. 2007. Automated segmentation of the optic nerve head from stereo color photographs using physiologically plausible feature detectors. Investigative Ophthalmology \& Visual Science 48(4):1665-1673. DOI: 10.1167/iovs.06-1081

Almazroa A, Burman R, Raahemifar K, Lakshminarayanan V. 2015. Optic disc and optic cup segmentation methodologies for glaucoma image detection: a survey. Journal of Ophthalmology 180972:1-28. DOI: 10.1155/2015/180972.

Aquino A, Gegúndez-Arias M, Marin D. 2010. Detecting the optic disc boundary in digital fundus images using morphological, edge detection, and feature extraction techniques. IEEE Transactions on Medical Imaging 29:1860-1869. DOI: 10.1109/TMI.2010.2053042

Cheng J, Liu J, Xu Y, Yin F, Wong DWK, Tan NM, Tao D, Cheng CY, Aung T, Won TY. 2013. Superpixel classification based optic disc and optic cup segmentation for glaucoma screening. IEEE Transactions on Medical Imaging 32(6):1019-1032. DOI: 10.1109/TMI.2013.2247770

Dashtbozorg B, Mendonça AM, Campilho A. 2015. Optic disc segmentation using the sliding band filter. Computers in Biology and Medicine 56:1-12. DOI:

10.1016/j.compbiomed.2014.10.009

Decencière E, Zhang X, Cazuguel G, Lay B, Cochener B, Trone C, Gain P, Ordonez-Varela JR, Massin P, Erginay A, Charton B, Klein JC. 2014. Feedback on a publicly distributed database: the Messidor database. Image Analysis \& Stereology 33(3):231-234. DOI: 10.5566/ias.1155.

Giachetti A, Ballerini L, Trucco E. 2014. Accurate and reliable segmentation of the optic disc in digital fundus images. Journal of Medical Imaging 1:1-11. DOI: 10.1117/1.JMI.1.2.024001.

Haleem MS, Han L, van Hemert J, Li B. 2013. Automatic extraction of retinal features from colour retinal images for glaucoma diagnosis: a review. Computerized Medical Imaging and Graphics 37:581-596. DOI: 10.1016/j.compmedimag.2013.09.005 
435 Lim G, Cheng Y, Hsu W, Lee ML. 2015. Integrated optic disc and cup segmentation with deep 436 learning. IEEE Conf. on Tools with Artificial Intelligence (ICTAI) 162-169. DOI:

437 10.1109/ICTAI.2015.36.

438 Lowell J, Hunter A, Steel D, Basu A, Ryder R, Fletcher E, Kennedy L. 2004. Optic nerve head 439 segmentation. IEEE Transactions on Medical Imaging 23(2):256-264. DOI:

$440 \quad 10.1109 /$ TMI.2003.823261

441 Lu S. 2011. Accurate and efficient optic disc detection and segmentation by a circular

442 transformation. IEEE Transactions on Medical Imaging 30:2126-2133. DOI:

443 10.1109/TMI.2011.2164261.

444 Marin D, Gegundez-Arias ME, Suero A, Bravo JM. 2015. Obtaining optic disc center and pixel 445 region by automatic thresholding methods on morphologically processed fundus images.

446 Computer Methods and Programs in Biomedicine 118(2):173-185. DOI:

$447 \quad 10.1016 /$ j.cmpb.2014.11.003

448

449

450

451

452

453

454

455

456

457

458

459

460

461

462

463

464

465

466

467

468
Mary MCVS, Rajsingh EB, Jacob JKK, Anandhi D, Amato U, Selvan SE. 2015. An empirical study on optic disc segmentation using an active contour model. Biomedical Signal Processing and Control 18:19-29. DOI: 10.1016/j.bspc.2014.11.003

Mendonça AM, Sousa A, Mendonça L, Campilho A. 2013. Automatic localization of the optic disc by combining vascular and intensity information. Computerized Medical Imaging and Graphics 37:409-417. DOI: 10.1016/j.compmedimag.2013.04.004.

Morales S, Naranjo V, Angulo J, Alcañiz M. 2013. Automatic detection of optic disc based on PCA and mathematical morphology. IEEE Transactions on Medical Imaging 32(4):786-796. DOI: 10.1109/TMI.2013.2238244

Ramakanth SA, Babu RV. 2014. Approximate nearest neighbour field based optic disk detection. Computerized Medical Imaging and Graphics 38(1):49-56. DOI:

10.1016/j.compmedimag.2013.10.007

Reza AW, Eswaran C, Hati S. 2009. Automatic tracing of optic disc and exudates from color fundus images using fixed and variable thresholds. Journal of Medical Systems 33(1):73-80. DOI: $10.1007 / \mathrm{s} 10916-008-9166-4$

Roychowdhury S, Koozekanani DD, Kuchinka SN, Parhi KK. 2016. Optic disc boundary and vessel origin segmentation of fundus images. IEEE Journal of Biomedical and Health Informatics 20(6):1562-1574. DOI: 10.1109/JBHI.2015.2473159

Sekar GB, Nagarajan P. 2012. Localisation of optic disc in fundus images by using clustering and histogram techniques. International Conference on Computing, Electronics and Electrical Technologies (ICCEET) 584-589. DOI: 10.1109/ICCEET.2012.6203911 
469 Suero A, Marin D, Gegundez-Arias ME, Bravo JM. 2013. Locating the optic disc in retinal 470 images using morphological techniques. Proc. International Work-Conference on Bioinformatics 471 and Biomedical Engineering (IWBBIO) 593-600.

472 University of Huelva. 2012. Expert system for early automatic detection of diabetic retinopathy

473 by analysis of digital retinal images. Available at http://www.uhu.es/retinopathy (accessed 29

474 May 2017).

475 Welfer D, Scharcanski J, Kitamura CM, Dal Pizzol MM, Ludwig LW, Marinho DR. 2010.

476 Segmentation of the optic disk in color eye fundus images using an adaptive morphological 477 approach. Computers in Biology and Medicine 40(2):124-137. DOI:

478 10.1016/j.compbiomed.2009.11.009.

479 Xu J, Chutatape O, Sung E, Zheng C, and Kuan PCT. 2007. Optic disc feature extraction via 480 modified deformable model technique for glaucoma analysis. Pattern Recognition 40:20634812076.

482 Yu H, Barriga ES, Agurto C, Echegaray S, Pattichis MS, Bauman W, Soliz P. 2012. Fast 483 localization and segmentation of optic disk in retinal images using directional matched filtering 484 and level sets. IEEE Transactions on Information Technology in Biomedicine 16(4):644 - 657. 485 DOI: 10.1109/TITB.2012.2198668

486 Zheng Y, Stambolian D, O'Brien J, Gee JC. 2013. Optic disc and cup segmentation from color 487 fundus photograph using graph cut with priors. Medical Image Computing and Computer488 Assisted Intervention 16(2):75-82. DOI: 10.1007/978-3-642-40763-5_10

489 Zhu X, Rangayyan RM. 2008. Detection of the optic disc in images of the retina using the Hough 490 transform. IEEE Conf. on Engineering in Medicine and Biology Society 3546-3549. DOI: 491 10.1109/IEMBS.2008.4649971.

492 Zilly J, Buhmann JM, Mahapatra D. 2017. Glaucoma detection using entropy sampling and 493 ensemble learning for automatic optic cup and disc segmentation. Computerized Medical 494 Imaging and Graphics 55:28-41. DOI: 10.1016/j.compmedimag.2016.07.012. 